\title{
REGULARITY OF VARIETIES IN STRICTLY PSEUDOCONVEX DOMAINS
}

\author{
FRANC FORSTNERIČ
}

\begin{abstract}
We prove a theorem on the boundary regularity of a purely p-dimensional complex subvariety of a relatively compact, strickly pse udoconvex domain in a Stein manifold. Some applications describing the structure of the polynomial hyll of closed curves in $C^{n}$ are also given.
\end{abstract}

\section{Introduction}

Let $X$ be a complex manifold, $M \subset X$ a connected $(2 p-1)$-dimensional submanifold of $X$ of class $C^{k}(k \geq 1, p \geq 1)$, and $A$ a closed complex subvariety of $X \backslash M$ of pure dimension $p$ such that $\bar{A} \subset A \cup M$. Then either $\bar{A}$ is a complex subvariety of $X$ or else there exists a closed subset $E \subset A$ of $(2 p-1)$ dimensional Hausdorff measure $\mathcal{K}_{2 p-1}(E)=0$ such that the pair $(A \backslash E, M \backslash E)$ is a $C^{k}$ submanifold with boundary $[2, p .190]$. In the second case $A$ has localiy finite $2 p$ dimensional volume in $X$, and $M$ can be oriented such that the pair $(A, M)$ satisfies the theorem of Stokes $[2, \mathrm{p} .192],[6],[8]$. Consequently $M$ is a maximally complex submanifold of $X$, i.e., the maximal complex subspace $\mathrm{T}_{z}^{C} M$ of the real tangent space $\mathrm{T}_{z} M$ to $M$ at $z$ has real codimension one in $\mathrm{T}_{z} M$.

There is a converse of this due to Harvey and Lawson [6]: If $X$ is a Stein manifold and $M$ is a closed, compact, maximally complex submanifold of $X$ of dimension $2 p-1(p \geq 2)$, then $M$ bounds (in the sense of currents) a purely $p$ dimensional complex subvariety $A \subset X \backslash M$, with boundary regularity as above.

We are interested in the boundary regularity of a purely p-dimensional complex subvariety of a relatively compact, strictly pseudoconvex domain $\Omega \subset X$ with $C^{2}$ boundary. We shall give a simple proof of the following

The work was supported in part by a grant from the Science Foundation of the Republic of Slovenia. 
Theorem 1. Assume that

(1) $X$ is a Stein manifold;

(2) $\Omega$ is a relatively compact, strictly pseudoconvex domain with $C^{2}$ boundary in $X$;

(3) $M$ is a closed $(2 p-1)$-dimensional submanifold of $X$ of class $C^{k}(p \geq 1$, $k \geq 2$ ) contained in the boundary $b \Omega$ of $\Omega$;

(4) $A$ is a purely p-dimensional complex subvariety of $\Omega$ such that $\bar{A} \subset$ $\subset A \cup M$, and $\bar{A}$ intersects every connected component of $M$.

Then there exists an open neighborhood $U$ of $M$ such that the pair $(A \cap U, M)$ is a $C^{k}$ manifold with boundary, and $\bar{A}$ intersects $b \Omega$ transversely in the set $M$.

Consequently $A$ has at most finitely many singularities in $\Omega$. The manifold $M$ is maximally complex, and its tangent space $\mathrm{T}_{z} M$ is not contained in the maximal complex tangent space $\mathrm{T}_{z}^{C} b \Omega$ to the boundary of $\Omega$ for any $z \in M$.

We obtain an interesting consequence concerning holomorphic convexity of closed curves. We shall state the result only for $X=\mathrm{C}^{n}$. Recall that the polynomially convex hull of a compact set $K \subset \mathrm{C}^{n}$ is

$$
\hat{K}=\left\{z \in \mathbb{C}^{n}:|f(z)| \leq \sup _{K}|f| \text { for all holomorphic polynomials } f\right\} .
$$

If $M$ is a rectifiable closed Jordan curve in $\mathbb{C}^{n}$, then either $M$ is polynomially convex, $M=\hat{M}$, or else $A=\hat{M} \backslash M$ is a purely one-dimensional analytic variety according to Wermer $[\mathbf{1 0}],[\mathbf{1 1}$, p.71], Stolzenberg [9], and Alexander [1]

Corollary 2. Let $\Omega$ be a bounded $C^{2}$ strictly pseudoconvex domain in $C^{n}$ with polynomially convex closure, and let $M$ be a simple closed curve of class $C^{k}, k \geq 2$, contained in the boundary of $\Omega$. If $M$ is not polynomially convex, then the one-dimensional complex variety $A=\hat{M} \backslash M$ has at most finitely many singularities.

Proof: Since $\bar{\Omega}$ is polynomially convex, $A$ is contained in $\bar{\Omega}$. Every point $p \in b \Omega$ is a peak point for $\Omega$, so the maximum principle implies that $A$ is contained in $\Omega$. Therefore the corollary follows from Theorem 1 .

We shall say that a submanifold $M \subset b \Omega$ of class $C^{1}$ is complex tangential at the point $z \in M$ if

$$
\mathrm{T}_{z} M \text { is contained in } \mathrm{T}_{z}^{C} b \Omega \text {. }
$$

Here, $\mathrm{T}_{z}^{C} b \Omega=\mathrm{T}_{z} b \Omega \cap \sqrt{-1} \mathrm{~T}_{z} b \Omega$. We shall say that $M$ is complex transverse at $z$ if it is not complex tangential.

Corollary 3. Let $\Omega \subset \mathbb{C}^{n}$ be as in Corollary 2. If $M \subset$ b $\Omega$ is a simple closed curve of class $C^{2}$ that is complex tangential at least at one point, then $M$ is polynomially convex.

Proof: If $M$ is not polynomially convex, Theorem 1 implies that the polynomial hull $\hat{M}=A \cup M \subset \bar{\Omega}$ is a complex variety with smooth boundary near 
every point $z \in M$, and $\hat{M}$ intersects $b \Omega$ transversely in $M$. This implies that $M$ is complex transverse in $b \Omega$ and the corollary follows.

Example. If $M$ is a simple closed $C^{2}$ curve in the sphere $\left\{z \in \mathbb{C}^{n}:|z|=1\right\}$ parametrized by the map $r(t)=\left(r_{1}(t), \ldots, \tau_{n}(t)\right)$ with nonvanishing derivative, and if

$$
\sum_{j=1}^{n} r_{j}^{\prime}(t) \overline{r_{j}(t)}=0
$$

for some value of the parameter t, then $M$ is polynomially convex

It seems rather surprising that a condition at one point of the curve guaranties its polynomial convexity, as long as the curve stays inside the given strictly pseudoconvex boundary.

\section{Remarks.}

1. Theorem 1 is stated in [2, p.203], but the proof given there does not appear to be complete.

2. If one knows already that $M$ is the boundary of $A=\hat{M} \backslash M$ in the sense of currents and if $p \geq 2$, then Theorem 1 is a special case of Theorem $10.3 \mathrm{in}$ $[6$, p.275].

3. In the case when $p=1$ and the variety $A$ is a proper holomorphic image of the unit disc $\Delta=\{z \in \mathbb{C}:|z|<1\}$, Theorem 1 follows from the more general results of Cirka $[3]$ concerning the regularity of one-dimensional complex varieties in the complement of a totally real submanifold of the ambient space.

4. In the case $p \geq 2$, Theorem 1 was proved by the author in [4]. Our new proof is simpler and includes the case $p=1$ when $M$ is a curve. We first show that the pair $(A, M)$ is a manifold with boundary in a neighborhood of each point $z \in M$ at which $M$ is complex transversal, i.e., the condition (1) fails. The proof in this case is the same as in [4]. The main difficulty in [4] was to show that $M$ can not be complex tangential at any point if it bounds a $p$ dimensional variety. In this paper we prove this by a very simple perturbation argument.

Acknowledgement. I wish to thank Josip Globevnik for several stimulating discussions on this subject.

\section{Proof of Theorem 1}

By the embedding theorem of fornæss and Khenkin $[7, \mathrm{p} .112]$ we may assume that $X=c^{n}$ and $\Omega$ is a strictly convex domain in $\mathcal{C}^{n}$.

It suffices to prove that each point $z^{0} \in \bar{A} \cap M$ has an open neighborhood $U$ such that the pair $(A \cap U, M \cap U)$ is a smooth manifold with boundary. We first prove this in the case when $M$ is complex transverse at $z^{0}$, i.e., condition (1) fails. This part of the argument is the same as in [4]. We include it for the convenience of the reader.

By an affine change of coordinates in $\mathbb{C}^{n}$ we may assume that 
(i) $z^{0}=0$,

(ii) $T_{0} b \Omega=\left\{\Re e z_{1}=0\right\}$ and $T_{0}^{C} b \Omega=\left\{z_{1}=0\right\}$, and

(iii) the domain $\Omega$ is contained in $\left\{\Re e z_{1}>0\right\}$.

Recall that $T_{0} M$ is a real $(2 p-1)$-dimensional subspace of $\left\{\Re z_{1}=0\right\}$ that is not contained in $\left\{z_{1}=0\right\}$. Thus the orthogonal projection of $\mathrm{T}_{0} M$ onto the $z_{1}$ axis is a real line, and the intersection $W=\mathrm{T}_{0} M \cap\left\{z_{1}=0\right\}$ has real dimension $2 p-2$.

We can choose a complex $(p-1)$-dimensional subspace $L$ contained in $\left\{z_{1}=\right.$ $=0\}$ such that the orthogonal projection $C^{n} \rightarrow L$ maps $W$ surjectively onto $L$. After a unitary change of coordinates $z_{2}, \ldots, z_{n}$ we may assume that $L=\left\{z_{1}=\right.$ $=z_{p+1}=\ldots=z_{n}=0$.

Let $\pi: C^{n} \rightarrow \mathbb{C}^{p}=\left\{z_{p+1}=0, \ldots, z_{n}=0\right\}$ be the orthogonal projection. Since $b \Omega$ is strictiy convex, we can find an open polydisc neighborhood $U=$ $=U^{\prime} \times U^{\prime \prime}$ of 0 in $\mathbb{C}^{n}$, with $U^{\prime} \subset C^{p}$ and $U^{\prime \prime} \subset \mathbb{C}^{n-p}$, such that $\pi: U \cap \bar{\Omega} \rightarrow U^{\prime}$ is a proper mapping. Our choice of $L$ implies that $\pi: T_{0} M \rightarrow \mathbb{C}^{p}$ is injective. Shrinking $U$ if necessary it follows that $\pi$ maps $M \cap U$ diffeomorphically onto a real hypersurface $\Gamma \subset U^{\prime}$ of class $C^{k}$ that splits $U^{\prime} \backslash \Gamma$ in two connected components $\Gamma^{+}$and $\Gamma^{-}$. Let $\Gamma^{+}$be the region contained in $\left\{\Re e z_{1}>0\right\}$. Since $M \cap U$ is contained in the strictly convex boundary $b \Omega \cap U$ and $C^{p} \times\{0\}$ contains the normal vector $(1,0, \ldots, 0)$ to $b \Omega$ at 0 , the projection $\pi(M \cap U)=\Gamma$ is hypersurface in $C^{p}$ which is strictly convex from the side $\mathrm{I}^{+}$, provided that the neighborhood $U$ is sufficientiy small.

Since $\pi: \bar{\Omega} \cap U \rightarrow U^{\prime}$ is proper and the set $(A \cup M) \cap U$ is closed in $U$, the restriction

$$
\pi:(A \cup M) \cap U \rightarrow U^{\prime}
$$

is also proper. The convexity of $\Gamma^{+}$along $\Gamma$ implies that $\pi(A \cap U)$ is contained in $\Gamma^{+}$according to the maximum principle. Hence the mapping

$$
\pi: A \cap U \rightarrow \Gamma^{+}
$$

is an analytic cover $[5$, p.101].

Denote by $s$ the number of sheets of this analytic cover, i.e., the number of points in the generic fiber. Notice that all sheets converge to the common edge $M$ as we approach $\Gamma$. We claim that this implies $s=1$. We only give a sketch of proof since the details can be found in [4].

Let $z=\left\{z^{\prime}, z^{\prime \prime}\right)$, where $z^{\prime}=\left(z_{1}, \ldots, z_{p}\right)$ and $z^{\prime \prime}=\left(z_{p+1}, \ldots, z_{n}\right)$. There is a linear function $w=w\left(z^{\prime \prime}\right)$ that separates points of $\pi^{-1}\left(z^{t}\right) \cap A \cap U$ for all points $z^{\prime} \in \Gamma^{+}$outside a proper complex subvariety $\sigma \subset \Gamma^{+}$. For each $z^{\prime} \in \Gamma^{+} \backslash \sigma$ we denote by $w_{1}\left(z^{\prime}\right), \ldots, w_{s}\left(z^{\prime}\right)$ the values of $w$ at the points of $\pi^{-1}\left(z^{\prime}\right) \cap A \cap U$. Let $P\left(w, z^{\prime}\right)$ be the polynomial in $w$ defined by

$$
P\left(w, z^{\prime}\right)=\prod_{j=1}^{s}\left(w-w_{j}\left(z^{\prime}\right)\right)=w^{s}+a_{1}\left(z^{\prime}\right) w^{s-1}+\cdots+a_{s}\left(z^{t}\right), \quad z^{\prime} \in \Gamma^{+} \backslash \sigma .
$$


Its coefficients $a_{j}\left(z^{t}\right)$ are bounded holomorphic functions on $\mathrm{\Gamma}^{+} \backslash \sigma$, so they extend to bounded functions on $\Gamma^{+}$. The discriminant $\delta\left(z^{\prime}\right)$ of $P\left(., z^{\prime}\right)$ is also a bounded holomorphic function on $\Gamma^{+}$since it is a polynomial expression in the coefficients $a_{j}$ of $P$. Recall that $\delta\left(z^{\prime}\right)=0$ if and only if $P\left(., z^{\prime}\right)$ has multiple roots.

If $s>1$, the hypothesis $\bar{A} \subset A \cup M$ implies that the nontangential boundary values of $\delta$ on $\Gamma$ equal zero almost everywhere since the different sheets of (2) converge together to $M$. This implies $\delta \equiv 0$ on $\Gamma^{+}$, a contradiction. Thus $s=1$ as claimed.

It follows that the projection (2) is a bijection, so $(A \cup M) \cap U$ is a graph of the form

$$
(A \cup M) \cap U=\left\{\left(z^{\prime}, f\left(z^{\prime}\right)\right): z^{\prime} \in \Gamma^{+} \cup \Gamma\right\} .
$$

Since $A$ is complex analytic and $M$ is of class $C^{k}$, it follows that $f$ is holomorphic on $\Gamma^{+}$and of class $C^{k}$ on $\Gamma$. Clearly $f$ is also continuous on $\Gamma^{+} \cup \Gamma$. The regularity theorem $[6, p .249]$ implies that $f$ is of class $C^{k}$ on $\Gamma^{+} U \Gamma$. This proves that $(A \cup M) \cap U$ is a $C^{k}$ manifold with boundary intersecting bn transversely.

It remains to show that the manifold $M$ is complex transverse at each point $z \in M \cap \vec{A}$ so that the first part of the proof applies. The following argument is considerably simpler than the one in [4], and it also applies in the case $p=1$.

Assume that the condition (1) is satisfied for some $z=z^{0} \in M \cap \bar{A}$. Let $\Lambda \subset \mathrm{T}_{z^{\circ}}^{C} b \Omega$ be the smallest complex subspace of $c^{n}$ containing $\mathrm{T}_{z^{\circ}} M$. Since $\mathrm{T}_{z^{\circ}} M$ is not a complex subspace, there is a vector $b \in A \backslash \mathrm{T}_{z^{\circ}} M$. We can choose a function $h$ of class $C^{2}$, supported on a neighborhood of $z^{0}$ in $C^{n}$, such that $\left.h\right|_{M} \equiv 0$, but the derivative of $h$ at $z^{0}$ in the direction $b$ is nonzero.

Let $\rho$ be a strictly convex defining function of class $C^{2}$ for $\Omega$, so $\Omega=\{z \epsilon$ $\left.\epsilon \mathbb{C}^{n}: \rho(z)<0\right\}$ and $d \rho \neq 0$ on $b \Omega$. If $\epsilon>0$ is sufficiently small, the domain

$$
\Omega_{\epsilon}=\left\{z \in \mathcal{C}^{n}: \rho(z)+\epsilon h(z)<0\right\}
$$

is of class $C^{2}$ and strictly convex. Fix such an $\epsilon$. Since $h$ vanishes on $M, M$ is contained in the boundary of $\Omega_{\epsilon}$. Thus we have $A \subset \hat{M} \subset \hat{\Omega}_{\varepsilon}=\bar{\Omega}_{\varepsilon}$, and the maximum principle implies $A \subset \Omega_{\mathrm{f}}$.

Our choice of $h$ implies that $T_{z^{\circ}} b \Omega_{\epsilon}$ does not contain $A$, so $T_{z^{\circ}}^{C} b \Omega_{\epsilon}$ does not contain $\mathrm{T}_{z^{\circ}} M$. This means that $M$ is complex transverse in $b \Omega_{\epsilon}$ at the point $z^{0}$. By the first part of the proof, with $\Omega$ replaced by $\Omega_{\epsilon}$, the set $\bar{A}$ is a local $C^{*}$ manifold with boundary $M$ near $z^{0}$.

We have proved that the pair $(A, M)$ is a local manifold with boundary near every point $z \in \bar{A} \cap M$. This implies that $\bar{A} \cap M$ is an open and closed subset of $M$. Since we assumed that $\bar{A}$ intersects every connected component of $M$, it follows that $\bar{A}=A \cup M$.

It remains to show that $\bar{A}$ intersects $b \Omega$ transversely. The restriction $\rho^{\prime}=\left.\rho\right|_{\bar{A}}$ of the plurisubharmonic defining function $\rho$ of $\Omega$ to $\bar{A}$ is a negative subharmonic 
function of class $C^{2}$ on the complex manifold with boundary $\bar{A}$. The Hopf lemma implies

$$
\rho(z) \leq-c \operatorname{dist}(z, M), \quad z \in A
$$

for some $c>0$. Here, dist denotes the Euclidean distance. Since $-\rho(z)$ is proportional to the distance of $z$ to $b \Omega$, we conclude that $\operatorname{dist}(z, M)$ is proportional to $\operatorname{dist}(z, b \Omega)$ for $z \in A$. Hence $\bar{A}$ intersects $b \Omega$ transversely at each point of $M$. Thus the condition (1) fails and $M$ is everywhere complex transverse.

\section{References}

1. H. ALEXANDER, Polynomial approximation and hulls in sets of finite linear measure in $C^{n}$, Amer. J. of Math. 93 (1971), 65-74.

2. E.M. ČIRKA, "Complex Analytic Varieties," (Russian) Nauka, Moskva, 1985.

3. E.M. ČIRKA, Regularity of boundaries of analytic sets, Mat. Sb. 117 (1982), 291-336; Math. USSR Sbor. 45 (1983), 291-335.

4. F. FORSTNERIČ, On the boundary regularity of proper holomorphic mappings, Annali Sc. Norm. Sup. Pisa Cl. Sc. Ser. IV, 13 (1986), 109-128.

5. R. GUNNING AND H. ROSSI, "Analytic Functions of Several Complex Variables," Prentice-Hall, Englewood Cliffs, 1965.

6. F.R. HARVEY AND H.B. LAWSON, On boundaries of complex analytic varieties I, Ann. of Math. 102 (1975), 223-290.

7. S.G. KRANTZ, "Function Theory of Several Complex Variables," John Wiley and Sons, New York, 1982.

8. N. SiBoNY, Quelques problemes de prolongement de courants en analyse complexe, Duke Math. J. 52 (1985), 157-197.

9. G. STOLZENBERG, Uniform approximation on smooth curves, Acta Math. 115 (1966), 185-198.

10. J. WERMER, The hull of a curve in $C^{n}$, Ann. of Math. 68 (1958), 550-561.

11. J. WeRmer, Banach Algebras and Several Complex Variables, SpringerVerlag, New York 1976.

Institut of Mathematics, Physics, and Mechanics Jadranska 19

YU-61000 Ljubljana, YUGOSLAVIA.

Rebat el 14 de Desembre de 1987 